\title{
Les années charnières
}

Depuis lacréation de médecine/sciences, en 1985, nous profitons du rite des voux pour nous pencher sur ce qui s'est passé dans l'année écoulée, la manière dont nous en avons rendu compte, et aussi sur nos projets. Le titre de ce texte, «Les années charnières", se réfêre non seulement au petit nombre d'années qui nous séparent du prochain millénaire, mais aussi à l'appréciation du mouvement de nos disciplines en biologie et médecine: tout a débuté, pratiquement rien n'est achevé (et d'ailleurs le mot "fin " n'a probablement pas plus de sens en Science qu'en Histoire), mais certaines perspectives se sont nettement clarifiées.

Au tableau d'honneur des grandes avancées de l'année, il faut sûrement mettre la compréhension des mécanismes de l'infection des cellules par le virus VIH et l'amélioration des traitements. D'ailleurs, «les années SIDA» sont non seulement emblématiques quant à leur signification et à leurs répercussions culturelles et sociales, au miroir cruel qu'elles offrent des inégalités croissantes d'accès à la santé entre pays riches et pays pauvres, mais aussi en tant que paradigme de l'accélération du progrès des connaissances et des techniques dans notre monde moderne.

En effet, voilà une maladie infectieuse, virale, découverte en 1981 ; en 1983, l'agent responsable est isolé ; une année plus tard, la séquence de son génome est connue. Après une période d'errance un peu incompréhensible, mais somme toute brève, on aboutit en 1994 à la vision juste d'une maladie comportant une intense réplication virale, compensée pendant longtemps par la réponse immunitaire et l'élimination des cellules infectées jusqu'à l'épuisement des capacités de renouvellement lymphocytaire de l'organisme. A partir de la connaissance des gènes viraux, on aboutit à la caractérisation structurale et fonctionnelle des protéines virales, notamment des protéases et de la transcriptase inverse et, 15 ans seulement après la découverte de l'affection, une polychimiothérapie fondée sur l'inhibition conjointe de ces protéines s'avère, sinon certainement curative, du moins très efficace, aboutissant même à des rémissions complètes apparentes. Ce résultat est non seulement remarquable en terme de rapidité de mise au point, il est même prodigieux, si on se rappelle qu'auparavant il n'existait pratiquement pas de thérapie antivirale vraiment efficace en dehors des analogues de nucléosides dans les infections à virus herpétique et des interférons dans les hépatites. Parallèlement, par la singuliè-

Axel Kahn, Rédacteur en chef, France re conjonction de recherches en virologie, immunologie et endocrinologie moléculaire, on découvre les co-récepteurs de l'infection, qui sont des récepteurs de chimiokines couplés aux protéines $\mathrm{G}$, et on établit l'une des bases de la résistance génétique à la maladie: une délétion dans le gène de l'un de ces co-récepteurs. Si on compare cette séquence à celle de tous les grands fléaux infectieux de l'humanité, où plusieurs centaines d'années se sont généralement écoulées entre la découverte d'une maladie et la compréhension de sa pathogénie, on ne peut qu'être stupéfaits... de la contraction du temps, mais aussi de ce que le sentiment dominant des citoyens soit, à l'inverse, celui d'une désespérante lenteur. Ce dynamisme de la recherche sur le SIDA nous réjouit d'autant plus que les chercheurs francophones, d'ailleurs souvent auteurs de médecine/sciences, y ont particulièrement brillé: citons, par exemple, les noms de Luc Montagnier à Paris, pour la découverte du virus; de Gilbert Vassart à Bruxelles, pour l'identification des co-récepteurs; et de Bernard Belleau à Montréal, pour ses travaux sur les inhibiteurs de la transcriptase inverse, principalement le $3 \mathrm{TC}$. 1996 aura également été l'année des maladies à prions... mais ici plus pour des raisons d'émoi public que scientifiques. En effet, si le passage à l'homme de l'agent infectieux de l'encéphalite bovine spongiforme est désormais quasi certain, bien des inconnues persistent sur la nature réelle de cet agent infectieux et sur l'importance de cette transmission.

Un troisième ensemble de faits marquants en 1996 est la conjonction - nous devrions même dire l'intégration - du champ de la biologie du développement à celui de la médecine humaine, bien illustré par les sagas du facteur Sonichedgehog (Shh) et des membres de la famille du TGF- $\beta$.

Le récepteur Patched de Shh est déficient dans le syndrome de Gorlin et dans des épithéliomas cutanés baso-cellulaires. Les récepteurs et des intermédiaires de la voie de transmission du signal des facteurs de type TGF- $\beta$ sont inactivés dans des cancers humains. La protéine effectrice de l'un de ces facteurs, Wnt, équivalent du facteur Wingless de drosophile, est la $\beta$-caténine (équivalente de Armadillo de drosophile), et elle joue probablement un rôle essentiel dans la cancérogenèse colique.

Une telle convergence nous réjouit évidemment, non seulement du fait de l'élégance des recherches qui ont contribué à l'illustrer mais aussi parce qu'elle justifie excellemment toute la démarche de médecine/sciences, démontrant que si nous n'existions pas - ce qu'à Dieu ne plaise - il faudrait nous inventer. Notre rôle est d'ailleurs non seulement de tenter d'informer tout en "mettant en perspective" la Science qui se fait, mais aussi d'amener à s'interroger sur les conséquences sociales et éthiques possibles de cette Science, à la lumière de son histoire. De ce point de vue, la génétique humaine, et son développement impétueux actuel dans le domaine du déterminisme des comportements, méritent d'être suivis avec une extrême attention, non seulement du fait de leur intérêt scientifique intrinséque, mais aussi des tentatives nombreuses de récupération par des idéologies anciennes qui ont déjà conduit, dans le passé, à l'exclusion, à l'eugénisme et au racisme.

Pour être à la hauteur de tous ces défis des «années charnières" que noustraversons, médeci$n e /$ sciences doit évoluer, dans un contexte difficile marqué par l'abondance croissante des résultats scientifiques - rendant plus nécessaire encore leur synthèse - et par les contraintes économiques que chacun connaît. Entre 1987 et 1996, nous avons été accompagnés dans notre évolution par l'équipe de l'éditeur John Libbey Eurotext, dirigée par Monsieur Gilles Cahn; nous tenons à manisfester ici notre renonnaissance à toute cette équipe avec laquelle notre revue s'est considérablement développée, multipliant par trois le nombre de ses abonnés et par deux le nombre de ses pages. Avec le soutien tutélaire de nos fondateurs, en France et au Québec, nous avons fait de notre $5^{\mathrm{e}}$ et de notre $10^{c}$ anniversaire de grands événements conviviaux et scientifiques. Demain, en application des règles gouvernant l'attribution des marchés publics, nous continuerons notre route avec un nouvel éditeur, la société Masson. Avec cet éditeur, nous souhaitons que médecine/sciences continue son chemin exigeant, alliant rigueur et profondeur, continuité et aptitude à l'adaptation à un mouvement des connaissances et de la société qui donne parfois le vertige. Pour être à la hauteur de ces exigences, nous savons pouvoir compter sur nos lecteurs et nos auteurs, en nombre croissant, auxquels nous demandons de nous accompagner par leur fidélité, leurs suggestions et leurs critiques.

Chères lectrices, chers lecteurs, au nom de toutes les équipes rédactionnelles de médecine/sciences, en France et au Québec, nous vous souhaitons une année 1997 éblouissante.

Michel Bergeron, Rédacteur en chef, Québec
N o) Or . 4 4 .
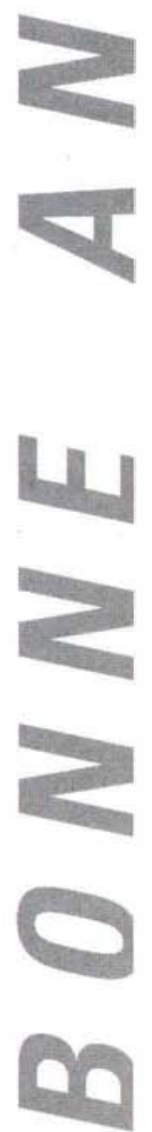


\section{La partie magazine de médecine/sciences a été réalisée grâce au concours de nombreux scientifiques dont la liste suit:}

André Adoutte

Patrick Aebischer

Djamila Aggoun

Marc Alizon

Patricia Amati

Brigitte Amiranoff

Yannick Andéol

Patrice André

Étienne Antoine

Tania Attié

Michèle Aubery

Johan Auwerx

Ségolène Aymé

Claude Bagnis

Ja-Hyun Baik

Sandrine Barbaux

Isabelle Baszanger

Hervé Bazin

Carole Beaumont

Jean-Paul Behr

Jean-Pierre Bellier

Yehezkel Ben-Ari

Patrick Bénard

Wail Benjelloun

Evelyne Benoit

Michel Bergeron

Catherine Bernard

Sylvie G. Bernier

Jérôme Bertherat

Jacques Bertoglio

Chrystelle Bidaud

Marc Billaud

Jean-Paul Blanchet

Jean-Marc Boeynaems

Eric Boitier

Dominique Bonneau

Jean-Pierre Bonvalet

Emiliana Borrelli

Thomas Bourgeron

Alexis Brice

Claude Brunel

Christine Bucharles

Elisabeth Bursaux

Bernard Calvino

Victoria Campuzano

Géraldine Cancel

Guy Cathala

Vincent Cavaillès

Christian Chabannon

Sophie Chappuis

Michel Chérel

Hervé Chneiweiss

Jean-Claude Chomel

Mireille Claustres

Karine Clément

Laurentiu Cocea

Didier Communi

Jean-Paul Concordet

J. Michael Conlon

Pierre Corvol

Mireille Cossée

Jean Costentin

Jean-Louis Couderc

Évelyne Coudrier

Yolaine Coulouarn

Yves Courtois

Guy de Thé

Françoise Degoul

Bernard Dastugue

Claude Debru
Sandrine Delannoy

Pierre Dellenbach

Erick Denamur

Herman Denis

Christelle Desbois

Jean Deutsch

Jean-Jacques Diaz

Vincent Di Martino

Betty Dodet

Bruno Doiron

Dominique Dormont

Pascal Dournaud

Jacques Drouin

Madeleine Duc Dodon

Antoine Dürrbach

Alain Duvallet

Jacques Elion

Jacques Epelbaum

Christophe Erneux

Marie-Odile Fauvarque

Marc Fellous

Laurent Ferradini

Jean-Louis Fischer

Nadine Forest

Thierry Forné

Alain Fournier

Didier Fradelizi

Philippe Froguel

Gérard Gacon

Imed Eddine Gallouzi

Robert Gardette

Raynald Gareau

Dominique Gauguier

Chantal Gauthier

Louis Gazzolo

Olivier Geneste

Pierre Gerlinger

Agnès Germont

Sraboni Ghose

Hélène Gilgenkrantz

Simone Gilgenkrantz

Anne Girardet

Bruno Giros

André M. Goffinet

Philippe Gorry

Claudine Grépin

Jean-Pierre Grünfeld

Michèle Guerre-Millo

Gaétan Guillemette

Jean-Gérard Guillet

Bernard Guy-Grand

Georg Haase

Alan Hall

Ahmed Hamliri

Alan Handyside

Jacques Hanoune

Louise Harel

Bruno Heyd

Najah Hmidi

Jean-Daniel Horisberger

Jean-Pierre Hugot

Edith Hummler

Georges Imbert

Pierre Jalinot

Jacques Jami

Marie-Claude Jaurand

Rajiv L. Joshi

Bertrand R. Jordan

Pierre Jouannet

Marie-Pierre Junier
Axel Kahn

Mimoun Kessabi

Karine Kindbeiter

Alain Kitzis

Michel Koenig

Blah Y.L. Kouassi

Alain Ktorza

Dominique Labie

Emmanuel Labourier

Hassan Lachkar

Catherine Lacombe

Didier Lacombe

Virginie Lacronique

Nathalie Lamarche

Paul Lang

Dominique Langin

Jocelyn Laporte

Mark Lathrop

Vincent Laudet

Michel Laurent

Pierre Laurent-Puig

Jean-Yves Le Gall

Hervé Le Guyader

Frédéric Lemaigre

Gilbert Lenoir

Frederick Libert

Isabelle Lihrmann

Vincent Lotteau

Stanislas Lyonnet

Jean-Jacques Madjar

Yves Malthièry

Jean-Louis Mandel

Patrice Mannoni

Cécile Marsac

Claude Matuchansky

Ken McElreavey

Igor Medina

Gilbert de Murcia

Josiane Ménissier de Murcia

Jean-Jacques Mercadier

Pierre-François Méry

Marc Mesnil

Jean-Claude Meunier

Mauro Mezzina

Alexandre Mignon

Delphine Mitanchez-Mokhtari

Catherine Mollereau

Ethel Moustacchi

Arnold Munnich

François Nantel

Fernando A. Navarro

Mona Nemer

Marc Nicolino

Jeanine Ohl

Marie-Odile Ott

Andras Paldi

Marc Parmentier

Andrea Pasini

Franck Pellestor

Christine Perret

Marc Peschanski

Hervé Philippe

Jean-François Picard

Jean-Yves Picard

Roberto Picetti

Michel Pinget

Stéphanie Possekel

Claude Poyart

Michel Pucéat

Pierre Ray
Tim Reid

Alfonso Represa

Claude-Agnès Reynaud

Pascal Reynier

Jacques Rochette

Béatrice Romagnolo

Christiane Rose

Ferdinand Rossi

Bernard C. Rossier

Jean-Luc Rossignol

Marina Roques

Jean-François Rouayrenc

Christian de Rouffignac

Guy Rousseau

Armin Ruf

Jacques Ruffié

Juan Ruiz

Evelyne Sage

Adolfo Saiardi

Régis Saladin

Rémi Salomon

Tarek Abdel Samad

Michel Samson

Alain Sarasin

Paolo Sassone-Corsi

Frédéric Saudou

Jean-Michel Sautier

Nathalie Schaerer-Uthurralt

Ernesto L. Schiffrin

Laurent Schild

Georg E. Schulz

Jean-Charles Schwartz

François Schweisguth

Bernard Sèle

François Sigaux

Denis Simonin

Bart Staels

Patrick Stragier

Jean Soulier

Thierry Soussi

Frédéric Tacnet

Joseph Tanzer

Jamal Tazi

Fabien Tell

Jacques Testart

Graziella Thiriet

Gilles Thomas

Hervé Tostivint

Aminata Touré

Patrick Trabal

Patrick Triadou

Eleni Tzavara

Thomas Valère

Gilbert Vassart

Guy Vassort

Hubert Vaudry

Didier Vieau

Cécile Viollet

Christophe Viret

Brigitte Viville

Stéphane Viville

Marion Wassef

Jean-Claude Weill

Daniel Widlöcher

Christiane Wittemer

Hiroshi Yamasaki

Gaël Yvert

Que tous ces collègues soient assurés de la reconnaissance des équipes de rédaction et des lecteurs de médecine/sciences.

La qualité de notre revue leur doit beaucoup. Nous leur présentons particulièrement tous nos vœux pour cette nouvelle année. 